\title{
Public Software Development Activity During the Pandemic
}

\author{
Vanessa Klotzman \\ University of California, Irvine \\ Irvine, California, USA \\ vklotzma@uci.edu
}

\author{
Farima Farmahinifarahani \\ University of California, Irvine \\ Irvine, California, USA \\ farimaf@uci.edu
}

\author{
Cristina Lopes \\ University of California, Irvine \\ Irvine, California, USA \\ lopes@uci.edu
}

\begin{abstract}
Background The emergence of the COVID-19 pandemic has impacted all human activity, including software development. Early reports seem to indicate that the pandemic may have had a negative effect on software developers, socially and personally, but that their software development productivity may not have been negatively impacted. Aims: Early reports about the effects of the pandemic on software development focused on software developers' well-being and on their productivity as employees. We are interested in a different aspect of software development: the developers' public contributions, as seen in GitHub and Stack Overflow activities. Did the pandemic affect the developers' public contributions and, of so, in what way? Method: Considering the data from between 2017 and till 2020, we study the trends within GitHub's push, create, pull request, and release events, and within Stack Overflow's new users, posts, votes, and comments. We performed linear regressions, correlation analyses, outlier analyses, hypothesis testing, and we also contacted individual developers in order to gather qualitative insights about their unusual public contributions. Results: Our study shows that within GitHub and Stack Overflow, the onset of the pandemic (March/April 2020) is reflected in a set of outliers in developers' contributions that point to an increase in activity. The distributions of contributions during the entire year of 2020 were, in some aspects, different, but, in other aspects, similar from the recent past. Additionally, we found one noticeably disrupted pattern of contribution in Stack Overflow, namely the ratio Questions/Answers, which was much higher in 2020 than before. Testimonials from the developers we contacted were mixed: while some developers reported that their increase in activity was due to the pandemic, others reported that it was not. Conclusion: In Github, there was a noticeable increase in public software development activity in 2020, as well as more abrupt changes in daily activities; in Stack Overflow, there was a noticeable increase in new users and new questions at the onset of the pandemic, and in the ratio of Questions/Answers during 2020. The results may be attributed to the pandemic, but other factors could have come into play.
\end{abstract}

\section{CCS CONCEPTS}

- General and reference $\rightarrow$ Empirical studies; Evaluation; Surveys and overviews.

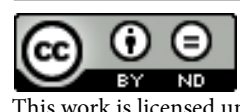

This work is licensed under a Creative Commons Attribution-NoDerivs International 4.0 License.

ESEM '21, October 11-15, 2021, Bari, Italy

(C) 2021 Copyright held by the owner/author(s).

ACM ISBN 978-1-4503-8665-4/21/10.

https://doi.org/10.1145/3475716.3475778

\section{KEYWORDS}

Pandemic, Developer activity, GitHub, Stack Overflow

\section{ACM Reference Format:}

Vanessa Klotzman, Farima Farmahinifarahani, and Cristina Lopes. 2021. Public Software Development Activity During the Pandemic. In ACM / IEEE International Symposium on Empirical Software Engineering and Measurement (ESEM) (ESEM '21), October 11-15, 2021, Bari, Italy. ACM, New York, NY, USA, 12 pages. https://doi.org/10.1145/3475716.3475778

\section{INTRODUCTION}

The COVID-19 pandemic hit the world in the beginning of 2020 and changed the aspect of daily living. With safety precautions in place since the start of the pandemic, many jobs and activities had to adapt to new routines and social distancing practices [12, 13, 17, 27]. The effect on the pandemic on assorted social and professional activities, including on software development, has been the target of intense study $[5,7,16,20,23,33,35,38,44]$. Software companies quickly adapted to new conditions by directing their employees to work from home $[8,18,34]$. This change in work practices clearly had effects in the software developers' activities [6, 25, 26, 39]. Some recent empirical studies document those effects more systematically in the context of proprietary software development [4, 17, 28]. In this paper, we describe our findings on whether there are any effects of the pandemic on public contributions to software development. On the one hand, being at home with more flexible schedules might have led more developers to do more work on open source projects $[2,30,40,41,46]$ and contribute more to questioning and answering community questions. On the other, job insecurity $[1,31]$ and personal disruptions brought by the pandemic might have swayed developers from getting distracted with any software development activities other than the ones at their jobs $[11,24]$.

By analyzing the data of two popular software development sites, we try to understand whether public software contributions ${ }^{1}$ have been impacted by the pandemic. Specifically, we formulate the following questions:

- RQ1: Were there any sudden changes in activity coinciding with the global stay-at-home orders? We consider the time frame of March/April 2020 for this purpose, as this was the period most of the world came under lock-down. We want to understand whether the initial shock led to a sudden contraction, expansion, whether it had a delayed impact, or whether it had no impact, in public contributions.

- RQ2: Were public contributions in the year 2020, in any way different from those between 2017-2019? Apart from possible outliers related to the onset of the pandemic, we want to

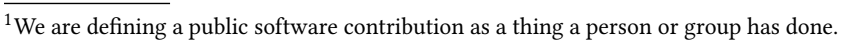


understand whether or not the year 2020 fell out of line with the trends of recent years.

- RQ3: Were the patterns of contribution disrupted in the year 2020? From public reports [19], we know that there are weekly and yearly patterns of contribution; for example, contributions decrease noticeably during the weekends and during the end of the year Holidays. We want to understand if those patterns held in 2020, and, generally, how the data compares on a yearly basis.

Our study unveils some insights of what happened to public software development contributions during the pandemic, what was different and what remained the same.

The rest of this paper is organized as follows: Section 2 elaborates on the dataset used in this study and the methodology followed to answer each of the research questions. In Section 3 we explain our findings for analyzing GitHub and in Section 4 we present the findings for Stack Overflow. In Section 5 we discussion our findings from both of these online developer communities. In Section 6 we discuss the body of research work related to ours, followed by a discussion on the threats to the validity of this study in Section 7 and conclusions in Section 8.

\section{DATA AND METHODOLOGY}

For GitHub, we used the GitHubArchive dataset, ${ }^{2}$ a project launched in 2012 that captures around 30 million GitHub events monthly. This dataset records the public GitHub timeline. The event types range from push and pull requests, new issues, comments, etc. As for Stack Overflow, its data is regularly published on the Internet Archive web site. ${ }^{3}$ This dataset contains information regarding daily activity of user registrations, user badges, posts (questions and answers), comments on posts, etc. For GitHub, we looked at the data from January 2017 until the end of 2020. For Stack Overflow, we looked at the data belonging to the period of January 2017 until the end of November 2020.

For this exploratory study, we answer each research question for various elements of activity in Stack Overflow and GitHub. We consider the number of pushes on GitHub. For Stack Overflow, we consider the number of new users, the number of questions asked and answers posted, the number of comments, and the number of votes given by users.

In most cases, the data we are looking at is non-stationary, meaning that it contains trending traits during the period in question. For example, the level of activity in GitHub has been steadily increasing over the past 4 years. For that reason, our analysis often includes transforming the raw data into differences between consecutive data points. This differencing technique eliminates background trends and makes the resulting time series approximately stationary [36]. For conciseness, we call this variation delta and show it with the $\Delta$ symbol. If $n$ is the number of observations on a time series and each observation in this interval is denoted by $O_{k}$ where $k \in\{0, . ., n-1\}$, then we compute $\Delta_{k}$ as:

$$
\Delta_{k}=O_{k}-O_{k-1}, k \in\{1, . ., n-1\}
$$

\footnotetext{
${ }^{2}$ http://www/gharchive.org

${ }^{3}$ https://archive.org/download/stackexchange
}

where $\Delta_{0}$ is undefined. We test whether the data is stationary or not with KPSS [36] and ADF [36] tests. The p_value threshold used for these tests is 0.05: with ADF test, for $p_{-}$value $>0.05$ then the series is non-stationary; with KPPS if $p_{\text {_ }}$ value $<0.05$ then the series is non-stationary.

Even though the data in both datasets is provided on a daily basis, for purposes of the different analyses, we look at the data using different time granularities. The following summarizes the statistical methodology we used for answering the research questions.

- RQ1 (Sudden changes in March/April 2020?). We answer this question by reducing the data to bimonthly ( 2 months) intervals. To understand whether the contributions in March/April 2020 were different, we investigate if this data point forms an outlier. We consider an outlier to be a data point that is 2 or more standard deviations away from the mean [48].

- RQ2 (Was 2020 different from recent past?). To answer this question, we consider the daily data for the number of contributions and split it into two datasets: (i) data concerning the years 2017 to 2019 and, (ii) data belonging to the year 2020. We then form the hypothesis:

$H_{0}$ : The 2020 data has the same distribution as the data of 2017-2019.

$H_{\alpha}$ : The 2020 data has does not have the same distribution as the data of 2017-2019.

We test this hypothesis with a two sample t-test [29] when the data has a normal distribution, or with Mann-Whitney test [14] when the data is not normally distributed [32]. We use the widely accepted $p \_v a l u e$ of 0.05 for rejecting $\mathrm{H}_{0}$.

- RQ3 (Yearly patterns of contribution). We answer this question by grouping the data into yearly bins and comparing the 2020 bin with all other bins. We compare several statistics, namely: the mean, the variance, and Shannon's entropy.

\section{GITHUB}

\subsection{Data Collection}

The GitHub Archive dataset contains a wide range of information regarding events, repository names, actors, payloads, and when the events were created. There are more than twenty event types. Associated with the event types, we have the metadata about the repository from which the user did the activity, when the activity happened, and the web hook payload. The event types range from push events and commenting to adding new members to a project. To understand the importance of these different types of events in the data we have available, we plotted the different event types in the dataset (see Figure 1). Push events are, by far, the ones with the highest frequency; more than $40 \%$ of the data for each year consists of push events (see Table 1). They are also highly correlated with most of the other events (see Table 2, Pearson correlation coefficients). As such, we present the details of the statistical analysis for push events only. For the other events, we provide only a brief summary at the end of this section.

\subsection{Analysis of Push Events}

In GitHub, a Push event happens when one or more commits are pushed to a repository branch or a tag. 


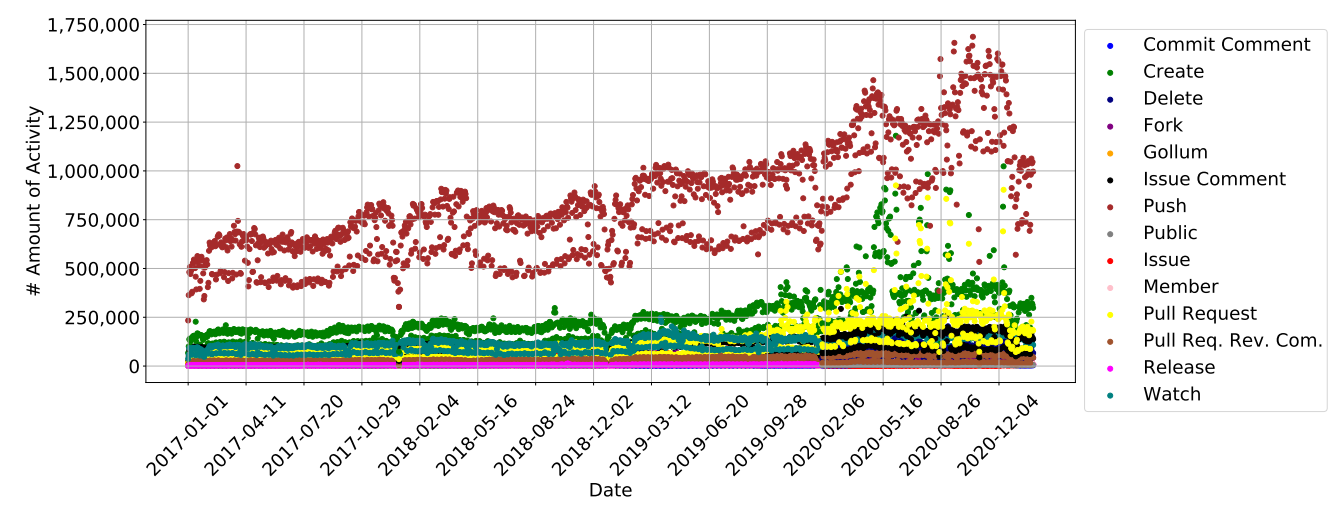

Figure 1: Frequency of GitHub activities: 2017 to 2020

Table 1: GitHub Dataset Statistics

\begin{tabular}{lrrrr}
\hline Activity Type & \multicolumn{1}{c}{2017} & \multicolumn{1}{c}{2018} & \multicolumn{1}{c}{2019} & \multicolumn{1}{c}{2020} \\
\hline Total & $412,243,377$ & $475,516,715$ & $605,392,987$ & $868,383,384$ \\
\hline Push & $218,630,527$ & $256,822,147$ & $313,322,116$ & $433,524,123$ \\
Create & $57,160,516$ & $66,774,841$ & $87,058,314$ & $145,329,073$ \\
Pull Request & $22,898,593$ & $27,604,776$ & $45,549,203$ & $84,571,677$ \\
Release & $1,515,452$ & $1,878,870$ & $2,375,740$ & $3,696,680$ \\
Fork & $11,744,988$ & $12,820,653$ & $16,476,611$ & $18,595,585$ \\
Issue Comment & $29,253,993$ & $31,108,096$ & $38,310,041$ & $54,114,952$ \\
Delete & $9,873,614$ & $12,193,730$ & $17,839,790$ & $28,439,209$ \\
Pull Req. Rev. Com. & $7,666,732$ & $8,577,616$ & $12,024,606$ & $16,563,300$ \\
Watch & $32,537,352$ & $37,068,153$ & $46,169,680$ & $49,186,313$ \\
Public & 408,286 & 493,803 & $1,643,883$ & $2,566,912$ \\
Member & $2,062,531$ & $2,174,329$ & $2,563,461$ & $3,089,564$ \\
Gollum & $2,586,673$ & $2,530,886$ & $2,522,818$ & $2,786,322$ \\
Issue & $14,922,351$ & $16,248,084$ & $18,833,721$ & $25,678,909$ \\
Commit Comment & 917,024 & 820,235 & $1,080,927$ & $2,773,242$ \\
\hline
\end{tabular}

Table 2: Correlation between Push Events and Other Events

\begin{tabular}{lc}
\hline & Push \\
\hline Create & 0.96 \\
Commit Comment & 0.858 \\
Fork & 0.918 \\
Gollum (Wiki action) & 0.545 \\
Issue Comment & 0.875 \\
Issues & 0.72 \\
Member & 0.734 \\
Public & 0.932 \\
Pull Request & 0.954 \\
Pull Request Review Comment & 0.846 \\
Release & 0.960 \\
Watch & -0.402 \\
Delete & 0.948 \\
\hline
\end{tabular}

RQ1: To determine whether there have been irregularities in terms of the number of Push events during the March/April 2020 time frame, we plotted the bimonthly (2 months) data, shown in

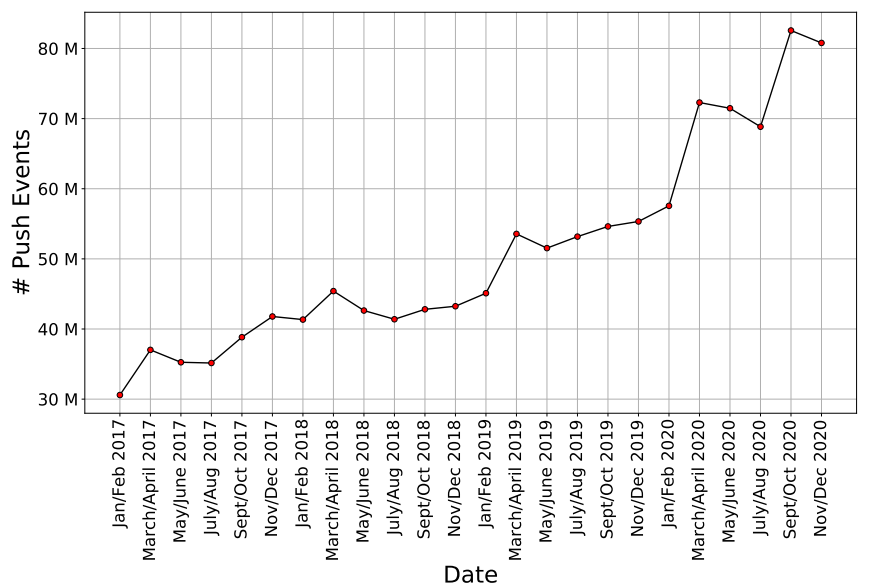

Figure 2: Bimonthly Number of Push Events on GitHub

Figure 2. As the figure shows, there has been a noticeable increase in the activity during the months of March/April.

This data is non-stationary - the visible increase over time is confirmed by an ADF test (p_value $=0.997)$ and a KPPS test ( $p \_v a l u e=0.032$ ). Therefore, we proceed with differencing it to make it suitable for statistical analysis. Figure 3 shows the data points after differencing, and the regression line on these data points. ${ }^{4}$ As the figure shows, the March/April data point has a significant difference with respect to previous data points. The mean of the $\Delta$ 's is $2,091,825$ and the standard deviation is $\approx 4,640,975$. The value of $\Delta$ during March/April 2020 is 14,730,122, which is more than 3 times the standard deviation of $\Delta$. This shows that this data point is an outlier.

RQ2: Similarly to the bimonthly data, the daily Push events time series is also non-stationary, showing an increase over time (KPSS p_value $=0.034$, ADF p_value $=0.1$ ). As such, we apply differencing. Figure 4 depicts the delta-version of the daily number of Push events. As the plot shows, we have noticeable daily outliers in the data, the

\footnotetext{
${ }^{4}$ The resulting data also shows traces of an increase, but it's much lower, so we interpret this as approximately stationary, and avoid a second-order differencing. Also, even though there are only 23 data points, the data is approximately normal.
} 


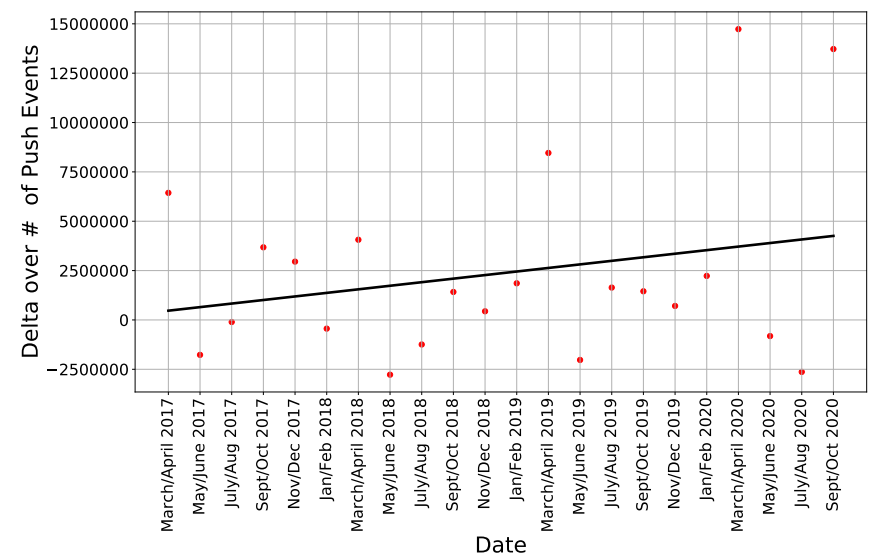

Figure 3: $\Delta$ over Bimonthly Number of Push Events

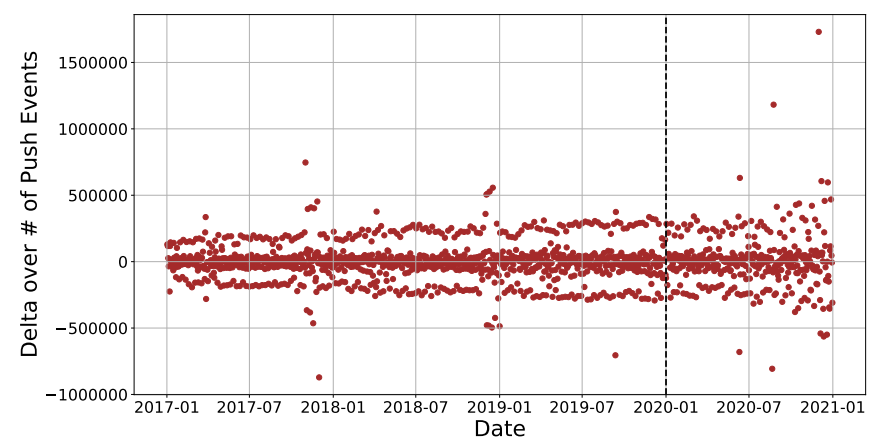

Figure 4: $\Delta$ over Daily Number of Push Events

largest ones being August 24, 2020 with a $\Delta$ count of 1,182,143, and December 1,2020 with a $\Delta$ count of $1,730,382$.

For RQ2, however, we aren't interested in outliers, but in the general distribution of the data for 2020 vs. the recent past. After applying the differencing technique, and removing the outliers $(<-500,000,>500,000)$, the mean of deltas for 2020 is 450 , while the mean of the previous years is 2,144 . The Shapiro-Wilk test identified that this data follows a normal distribution. The t-test shows $p-$ value $=0.07$, meaning that the difference in the means is not statistically significant. Therefore we do not have evidence to support the hypothesis that 2020 was different than the recent past.

RQ3: To answer RQ3, we split the data prepared in RQ2 into four different years, and compare some statistics related to variability, shown in Table 3 . These differences in means are not statistically significant ( $\mathrm{p}$ values $>0.05$ ).

As noted before [19], there are weekly cycles in this data, wherein contributions decrease on the weekends. This can be seen in the scatter plot of Figure 4, which embodies two visually distinct groups of data points, and outer one and an inner one. Since we are dealing with daily deltas, the outer group (larger increases and decreases) corresponds to the variations that happen Sundays to Mondays and Fridays to Saturdays. On visual inspection, it appears that the data for 2020 is more scattered than for previous years.
Table 3: RQ3: Statistics of $\Delta$ over Daily \# Push Events

\begin{tabular}{lrrrr}
\hline Year & 2017 & 2018 & 2019 & 2020 \\
\hline Mean & 753 & 4352 & 1329 & 450 \\
Var & $1.24 \times 10^{8}$ & $1.71 \times 10^{10}$ & $2.14 \times 10^{10}$ & $2.39 \times 10^{10}$ \\
Entropy & 0.72 & 0.75 & 0.80 & 0.85 \\
Outliers & 2 & 4 & 1 & 10 \\
\hline
\end{tabular}

Table 4: Stack Overflow Dataset statistics

\begin{tabular}{lcrrrr}
\hline & \multirow{2}{*}{ Users } & \multicolumn{2}{c}{ Posts } & \multirow{2}{*}{ Votes } & \multicolumn{1}{c}{ Comments } \\
\cline { 3 - 4 } & & Questions & Answers & & \\
\hline Total & $7,126,703$ & $7,945,781$ & $10,177,940$ & $86,404,248$ & $29,857,733$ \\
2017 & $1,728,421$ & $2,124,351$ & $2,913,396$ & $23,223,687$ & $8,669,683$ \\
2018 & $1,646,531$ & $1,901,338$ & $2,563,399$ & $22,244,375$ & $7,706,953$ \\
2019 & $1,721,658$ & $1,806,283$ & $2,410,316$ & $21,267,514$ & $6,905,108$ \\
2020 & $2,030,093$ & $2,113,809$ & $2,290,829$ & $19,668,672$ & $6,575,989$ \\
\hline
\end{tabular}

We investigate this observation by looking at the variance and entropy for each year, shown in Table 3. Both statistics show an increase over the past 4 years. An increase in variance means that the daily deltas have been getting increasingly farther from the mean. The increase in variance from 2019 to 2020 is aligned with, and even smaller, than previous increases. This is one signal that 2020 was not special with respect to variance. An increase in entropy means that the daily deltas have become more unpredictable. The entropy for 2020 is higher than those of previous years, but there was also a similar jump in entropy from 2018 to 2019. Again, 2020 does not seem special.

\subsection{Other Events}

We performed statistical analysis of all types of events shown in Table 2 . Not surprisingly, the findings for the events that are strongly correlated to push events $(>0.8)$ are similar to the findings unveiled in the previous subsection, for all three research questions. However, for the events that are not so strongly correlated with push events, namely the gollum, member, issue and watch events, the findings are different. Specifically, these do not have any outliers in 2020. The data points are stationary, and there is no presence of a trend. Interestingly, the outliers for watch and issue events occur primarily in 2019. This means that Wiki activities, project memberships, the number of issues opened/closed, and the amount of watch actions did not have any observable disruptions in 2020.

\section{STACK OVERFLOW}

\subsection{Data Collection}

As mentioned earlier, we considered the data from January 2017 until the end of November $2020^{5}$. For this analysis, we are looking at the number of new users joining Stack Overflow and number of posts (questions and answers), comments and votes; the timestamp fields associated with the creation of each of these entities can

\footnotetext{
${ }^{5}$ The Stack Overflow dataset was not yet published for December 2020 when the analysis was done. So we considered Stack Overflow data until the end of November 2020 .
} 


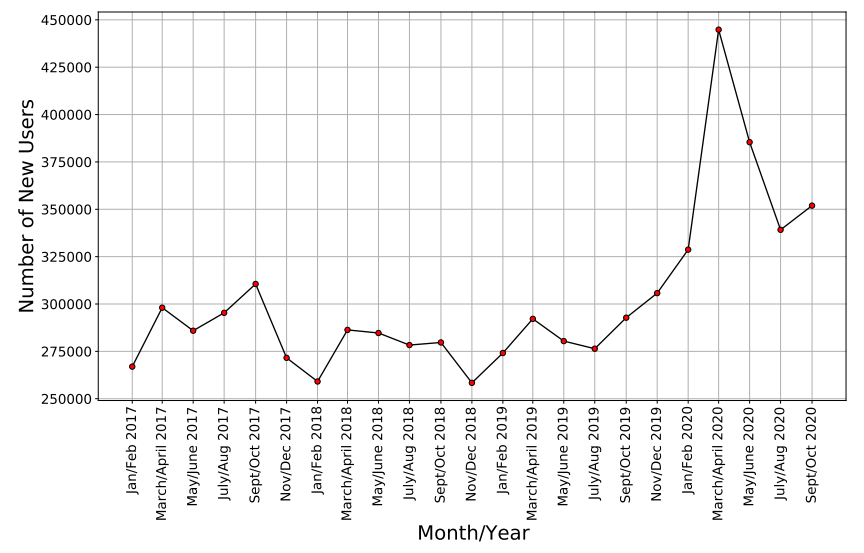

Figure 5: Bimonthly \# of New Users on Stack Overflow

help us in understanding the activity patterns on Stack Overflow. Table 4 provides an overall picture on the number of these entities for the period of interest. For each entity (e.g. new users) we present the total number for the period of 2017-2020 in the first row. The following rows show how many of the reported total numbers fall into each year. As the table shows, there have been more than 7 millions users joining Stack Overflow since the beginning of the 2017, where the year 2020, has had the maximum number of users joining Stack Overflow compared to other years. Nearly 8 million questions were asked in the period of 2017-2020 and the number of answers posted on Stack Overflow has passed 10 million. Both questions and answers have had the maximum number in year 2017. Users have posted more than 86 million votes in total in the period of four years and we observe a descending trend on the number of votes from 2017 toward 2020. Finally, $\approx 30$ million comments have been posted in total and, similar to number of votes, a descending trend has happened from 2017 toward 2020.

\subsection{Analysis of Stack Overflow Events}

In order to understand the patterns of activity on Stack Overflow, we analyzed each of the entities described in Section 4.1 with respect to each of the research questions.

4.2.1 Users. For each user, we have the date and time they joined Stack Overflow. Therefore, we can analyze the number of new users for our dates of interest.

$R Q 1$ : To understand whether there has been any irregularities in terms of the number of new users who joined Stack Overflow during March/April 2020, we plotted the number of new users in bimonthly intervals in Figure 5. The X-axis points to the bimonthly intervals and the Y-axis points to the number of new users. As the figure shows, there has been a sharp increase in the number of new users during March and April 2020.

This data is non-stationary (KPSS $p \_v a l u e=0.1, \mathrm{ADF} p \_v a l u e=$ $0.37)$. Therefore, we moved forward with making the data stationary by differencing and computed the $\Delta$ values. Figure 6 shows the computed $\Delta$ values and a regression line fit on them. As the plot shows, the $\Delta$ in March/April 2020 has a considerable distance from the fitted line. The mean of this data is $\approx 3,862$ and the standard

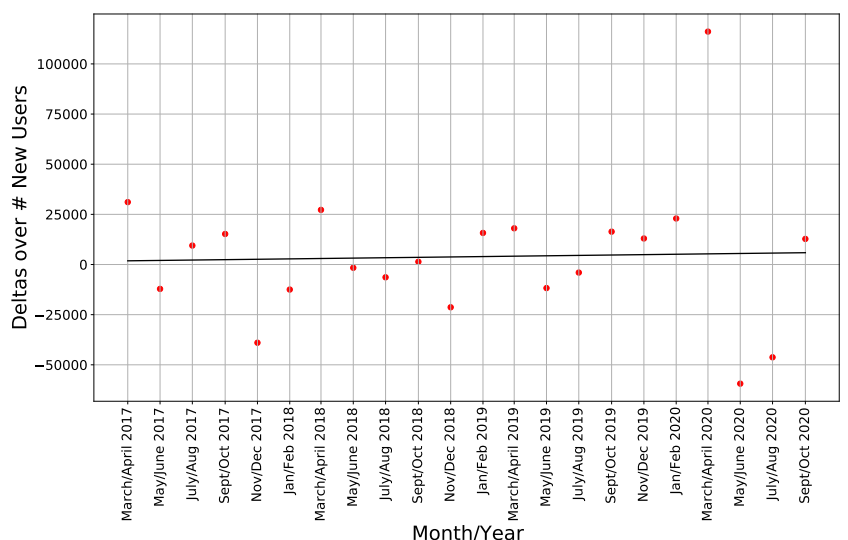

Figure 6: $\Delta$ over Bimonthly \# of New Users on Stack Overflow

deviation is $\approx 34,522$. The value of $\Delta$ in March/April 2020 is 116,110 which is $\approx 3.25$ times the standard deviation, satisfying our threshold of 2 . Hence, we can conclude that, according to the criteria defined for RQ1, March and April 2020 witnessed a sudden increase of new users in Stack Overflow.

RQ2: After breaking down the number of new user account creations into daily cycles, we tested this data with KPSS ( $p \_$value $=$ 0.01 , thus non-stationary) and $\mathrm{ADF}$ ( $p \_$value $=0.008$, thus stationary) tests. Since one test reports a non-stationary data, we applied differencing to get a stationary data for our analysis. Figure 7 depicts the daily number of new users after differencing. As the plot shows, there are a number of datapoints showing very big differences on this data, with the biggest one pointing to a difference of 6,000. We looked at this datapoint and it belongs to March 31, 2020; this shows that on March 31, 2020 there has been a record number of new users joining Stack Overflow over the past four years: the raw daily data shows that the actual number of users who joined Stack Overflow was more than 16,600 on this day. In order to continue our analysis, we removed such outliers from this data to prevent them from affecting the results of further statistical analysis: we removed the rows with $\Delta>3000$ and $\Delta<-3000$, resulting in eliminating 5 datapoints.

We then examined the normality of data with Shapiro Wilk test which reported a non-normal distribution. We then splitted data to two portions of 2017 to 2019 and 2020: the mean of $\Delta$ 's on 2020 data is $\approx-28.78$ and on $2017-2019$ data is $\approx-1.35$ which does not show a big margin of difference given the range of $\Delta$ values (-3000 to 3000). We then ran Mann Whitney test to test the hypothesis pertaining to RQ2. The test reported $p \_v a l u e=0.222$ which fails to reject the null hypothesis meaning that the two sets of data follow the same distribution. A close look at Figure 7 also attests these results as data before and after 2020 (except for a few outliers that we filtered out) follows the same distribution.

RQ3: To answer RQ3, we split the data prepared for RQ2 into four bins of: 2017, 2018, 2019 and 2020. We first compared the distribution of 2020 data with each of the other years using Mann Whitney test. The $p \_v a l u e$ reported by this test for comparing year 2020 with 2017 was 0.193 , with 2018 was 0.243 and with 2019 was 0.369 . Since all p_values are more than 0.05 , the distribution of 


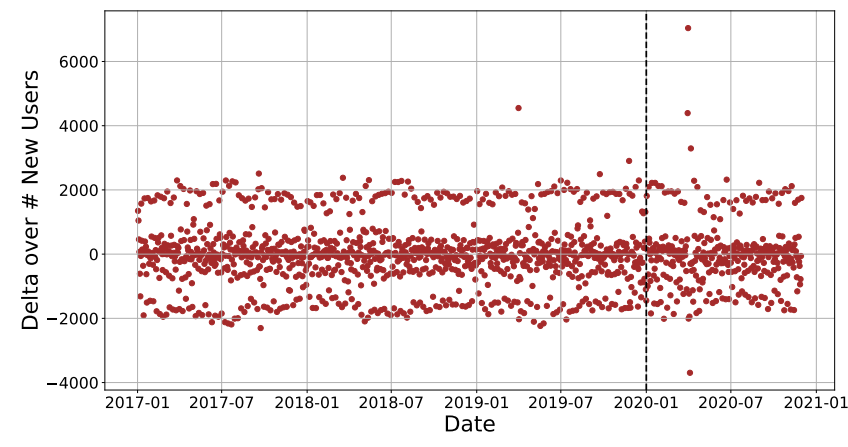

Figure 7: $\Delta$ over Daily \# of New Users on Stack Overflow

Table 5: RQ3: Statistics of $\Delta$ over Daily \#New Users

\begin{tabular}{lrrrr}
\hline Year & 2017 & 2018 & 2019 & 2020 \\
\hline Mean & 0.31 & 1.7 & -6.09 & -28.78 \\
Var & $1,020,889$ & 928,276 & $1,031,910$ & 892,551 \\
Entropy & 0.87 & 0.85 & 0.84 & 0.87 \\
Outliers & 0 & 0 & 1 & 4 \\
\hline
\end{tabular}

data in 2020 is no different than any other year. We also computed the statistical values for each year: Table 5 provides the values for mean, variance and entropy for the $\Delta$ values in each year. As the table shows, given the range of data, means of all years are close to each other and variances also have close values. The entropy values show that the level of uncertainty in the $\Delta$ values is very close for all years, ascertaining our conclusion that the distribution of number of new users joining Stack Overflow in 2020 has been similar to each of the previous years.

The main difference here is in the number of outliers. The year 2020 contains 4 out of the 5 total outliers since 2017. Outliers represent unusual increases/decreases on the number of new users on any two consecutive days. Interestingly, unlike the GitHub Push events, the 4 outliers in Stack Overflow in 2020 all fall within the March/April period - the onset of the pandemic.

4.2.2 Posts. As mentioned earlier, posts on Stack Overflow can either be questions or answers. Figure 8 shows the number of posts on Stack Overflow per month, categorized by questions (blue dotted line) and answers (orange solid line). As the plot shows, there has been a sudden increase in both the number of questions and answers at the beginning of the pandemic (mostly in the month of April 2020). According to this plot, the number of answers has always been greater than the number of questions but this gap became very narrow in the year 2020, and, in particular, in the months of March and April, we see a very small margin. The difference between the number of questions and the number answers for each year is also observable from Table 4. According to this table, the difference between the number of answers and the number of questions posted on Stack Overflow is the smallest in the year 2020: the number of answers is greater than the number of questions by a margin of only 177,020; where in 2017 for example, this difference is over 700,000. This pattern can tell us that although user engagement related

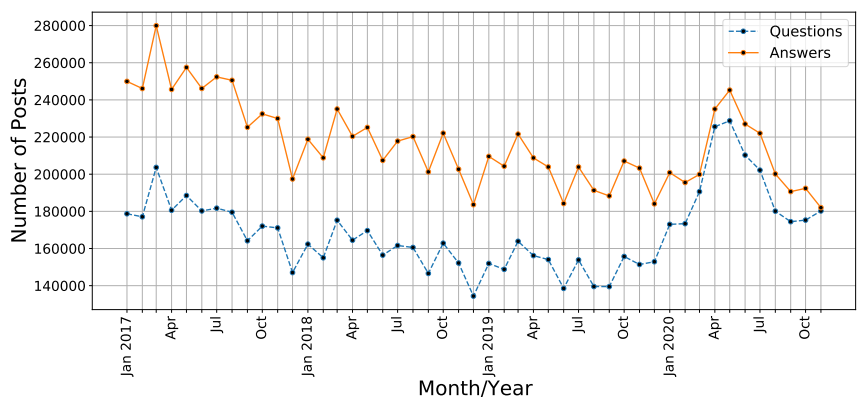

Figure 8: Monthly Number of Posts on Stack Overflow

to both questions and answers has increased in the beginning of the pandemic, the increase is more prominent with the number of questions.

In the rest of this section, we analyze questions and answers, separately, as well as the ratio Questions/Answers, with respect to each of the research questions.

Questions. The analysis of the number of questions asked on Stack Overflow is as follows.

RQ1: After splitting the questions data into bimonthly intervals, we tested this data with KPSS ( $p \_v a l u e=0.1$ thus, stationary) and $\mathrm{ADF}$ ( $p \_$value $\approx 0.01$, thus stationary) tests. Since both tests reported a stationary data, we moved forward with analyzing the data as is. Figure 9 shows the number of questions posted on Stack Overflow on a bimonthly interval with a regression line fitted on this data. As the plot shows, the March/April 2020 data point has a considerable distance from this line. The mean of this data is calculated as $\approx 337,633$ and standard deviation is $\approx 40,354$ and March/April data point has a value of 416,194 which is $\approx 2$ Standard deviations far from the mean. As a result, we can conclude that March/April 2020 had a considerable increase in the number of questions posted on Stack Overflow.

An interesting observation here is that as Figure 9 shows, the May/June 2020 datapoint also has a considerable distance from the regression line. The number of questions at this point is $\approx 2.5$ Standard deviations far from the mean, marking it as an outlier too.

RQ2: After breaking down the number of questions asked on Stack Overflow into daily intervals, we tested whether this data is stationary. KPSS test returned a $p \_v a l u e$ of 0.01 and ADF test returned a $p \_v a l u e$ of $\approx 0.15$, therefore, the data is not stationary. We applied differencing to convert it to stationary data for further analysis. Figure 10 shows the scatter plot of computed deltas after this process. As the plot shows, while a majority of data is centered in the interval of $[-1000,1000]$, there are two other sets of data points centered on top $(>2000)$ and at the bottom $(<-2000)$ of this plot. We looked more closely at these data points and it turned out that the ones $>2000$, showing a sudden increase on the number of questions, point to Mondays, marking the beginning of work days. On the other side of the spectrum, the data points that have values $<-2000$ point to Saturdays, marking the start of the weekends.

To answer RQ2, we continued by assessing the normality of this dataset; Shapiro Wilk test reports the data to not be following a normal distribution; therefore, after splitting data into two bins of 


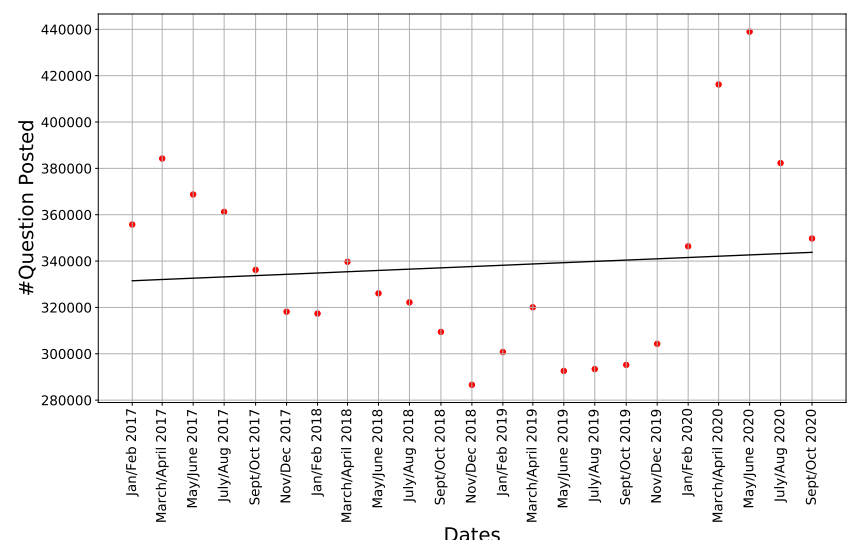

Figure 9: Bimonthly Num. of Questions on Stack Overflow

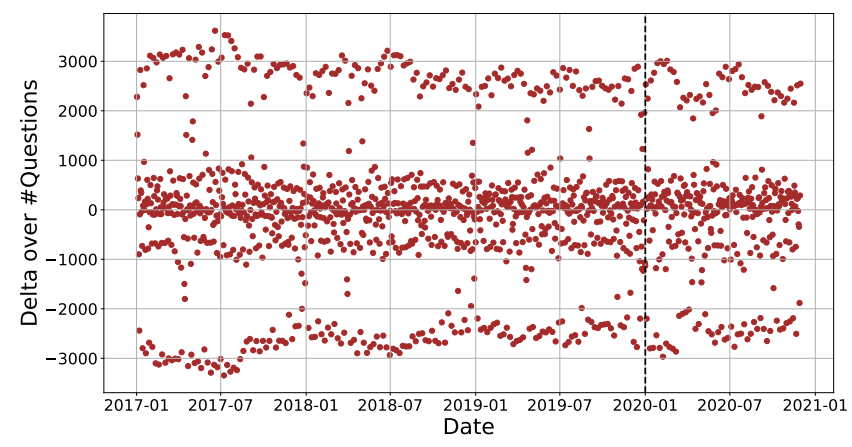

Figure 10: $\triangle$ over Daily Num. of Questions on Stack Overflow

2020 data and 2017-2019 data, we ran Mann Whitney test to test our hypothesis that these two are significantly different. The test reported a $p \_v a l u e$ of 0.168 , and therefore, failing to reject the null hypothesis, concluding that 2020 data is no different than the data before that. Also, the mean of $\Delta$ 's on $2017-2019$ data is $\approx 1.4$ and on 2020 data is $\approx 10.5$, which given the range of $\Delta$ values, is close.

RQ3: After splitting the daily $\Delta$ values of the number of questions to bins of 2017,2018, 2019 and 2020, we tested whether the distribution of 2020 data is different to that of any of the other years. The Mann Whitney test returned $p \_v a l u e=0.206$ for comparison with $2017, p \_v a l u e=0.164$ for comparison with 2018 and $p \_v a l u e=0.279$ for comparison with 2019. All $p \_v a l u e s$ are greater than 0.05 and therefore, there is no difference between the of data of 2020 with any other years. The statistical values presented for each year in Table 6 also support this conclusion: given the range of delta values (from Figure 10), the mean values are close. The variance of data in year 2020 has a value between the variance of 2019 and the variance of 2018. The entropy value for all years is also in a range of 0.78 to 0.81 meaning that the level of uncertainty was pretty similar in all these years. Hence, we can conclude that the number of questions in 2020 is not different than any other years.

Answers. The analysis over the number of answers is as follows. RQ1: The ADF test reports the answers' bimonthly counts to be non-stationary ( $p \_$value $\approx 0.17$ ) and KPSS test reports it to be
Table 6: RQ3: Statistics of $\Delta$ over Daily \# Questions

\begin{tabular}{lrrrr}
\hline Year & 2017 & 2018 & 2019 & 2020 \\
\hline Mean & -0.25 & 2 & 2.42 & 10.5 \\
Var & $2,571,849$ & $2,096,831$ & $1,873,660$ & $1,898,210$ \\
Entropy & 0.79 & 0.78 & 0.81 & 0.81 \\
Outliers & 0 & 0 & 0 & 0 \\
\hline
\end{tabular}

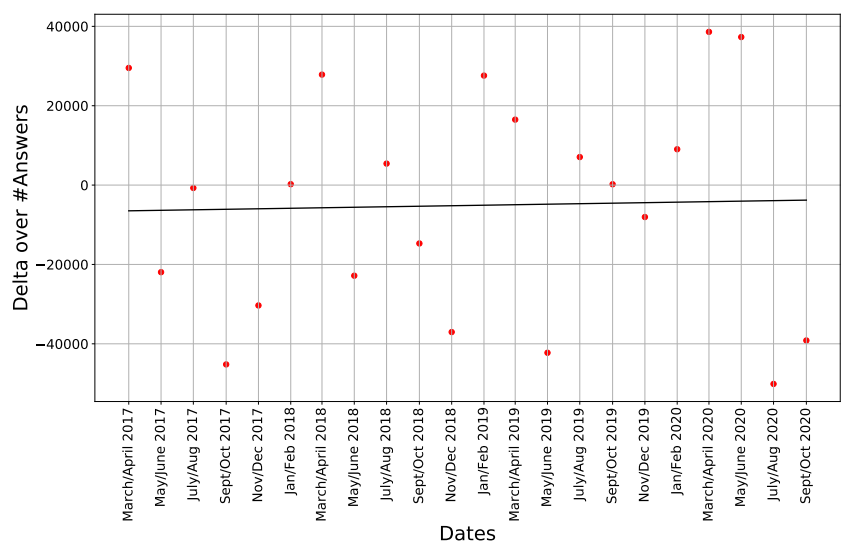

Figure 11: $\Delta$ over Bimonthly \#Answers on Stack Overflow

stationary $\left(p \_v a l u e=0.1\right)$. Since one test reports data to be nonstationary, we applied differencing on this data. Figure 11 shows the deltas of the number of answers in bimonthly intervals and a regression line fitted on this data. As the plot shows, the March/April 2020 data point is a distanced point from this line, however, there are several other distant data points as well, such as Sep/October 2017 and May/June 2020. To understand whether March/April 2020, which has a value of 38,611 , forms an outlier, we calculated mean and standard deviation over this data: mean is $\approx-5,139$ and standard deviation is $\approx 28,108$. March/April 2020 data point is 1.6 times standard deviation far from the mean. Although very close, this is less than our defined threshold of 2 and is not an outlier.

$R Q 2$ : We started by breaking down the number of answers posted on Stack Overflow into daily intervals and testing whether it is stationary. KPSS test reported a $p \_v a l u e$ of 0.01 and ADF test reported a $p \_v a l u e$ of $\approx 0.04$. Since one test reports data to be non-stationary (KPSS), we moved forward with differencing to make this data stationary. Figure 12 shows the scatter plot of the data after differencing. The same weekend/workday pattern of spreading $\Delta$ 's is also observable in this plot, however, the gap between weekends and workdays becomes narrow toward the recent years.

According to Shapiro Wilk test, this data does not follow a normal distribution, so we use Mann Whitney test to test our hypotheses pertaining to RQ2: the test reports a p_value of 0.328 which is greater than 0.05 , failing to reject $H_{0}$. This means that number of answers in 2020 and in 2017-2019 have the same distribution. Also, mean of $2017-2019$ data is $\approx 1.5$ and mean of 2020 data is $\approx 4.9$, with two values being close, our conclusion becomes more certain.

RQ3: Following the procedure for RQ3, we separated the daily $\Delta$ values into bins of 2017, 2018, 2019 and 2020 and compared the 


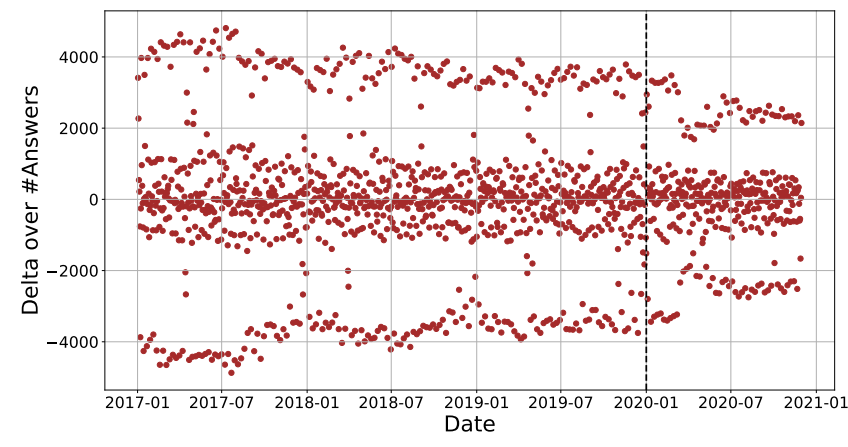

Figure 12: $\Delta$ over Daily \#Answers on Stack Overflow

Table 7: RQ3: Statistics of $\Delta$ over Daily \# Answers

\begin{tabular}{lrrrr}
\hline Year & 2017 & 2018 & 2019 & 2020 \\
\hline Mean & -0.25 & 3.2 & 1.5 & 4.9 \\
Var & $5,047,800$ & $3,954,864$ & $3,546,847$ & $2,037,858$ \\
Entropy & 0.82 & 0.8 & 0.82 & 0.82 \\
\hline
\end{tabular}

distribution of $\Delta$ in 2020 with each of the other years using Mann Whitney test, which returned $p \_v a l u e=0.28$ for comparison with $2017, p \_v a l u e=0.339$ for comparison with 2018 and $p \_v a l u e=0.46$ for comparison with 2019. Similar to what we saw for questions, all $p \_v a l u e s$ are greater than 0.05 and therefore, the distribution of data in 2020 is similar to other years. We also computed the other necessary statistical values, listed in Table 7 . The mean values for all years are close and the variance of data in 2020 is the least among other years. Based on the entropy values, the uncertainty level of the data pertaining to the number of answers is very close for all years. As a result of all this, we conclude that the pattern of number of answers posted on Stack Overflow in 2020 has been similar to the years before it.

Questions/Answers. Figure 8 suggests that the year 2020 was particularly different in terms of the ratio of questions/answers $(\mathrm{Q} / \mathrm{A})$. In here, we make a statistical analysis of that ratio.

$R Q 1$ : Similarly to the other elements, we created a bimonthly time series with the deltas of the ratio $\mathrm{Q} / \mathrm{A}$ which is shown in Figure 13. Here, too, the outliers are quite visible - the two data points corresponding to Jan/Feb 2020 and March/April 2020. Interestingly, the the first outlier was before March. This may suggest activities originating in China, which went on lock down around that time. However, we cannot tell for sure. In any case, these two data points are 2 or more standard deviations away from the mean $($ Mean $=0.008906$, Std $=0.029193$; Jan/Feb:0.0794; Mar/Apr: 0.0744 ), marking them outliers according to our criteria.

RQ2: The mean of Q/A data for the period of 2017 to 2019 is 0.74 and and mean of 2020 data is 0.92 . The data is not normal so we ran Mann Whitney test to test RQ2 hypothesis, which returned p_value $<0.05$, meaning that these two data series have different distributions.

$R Q 3:$ The means, variances, entropy and outliers are summarized in Table 8. The Mann Whitney test for comparing $2020 \mathrm{Q} / \mathrm{A}$ data with all other years returned $p \_v a l u e<0.05$, suggesting that the

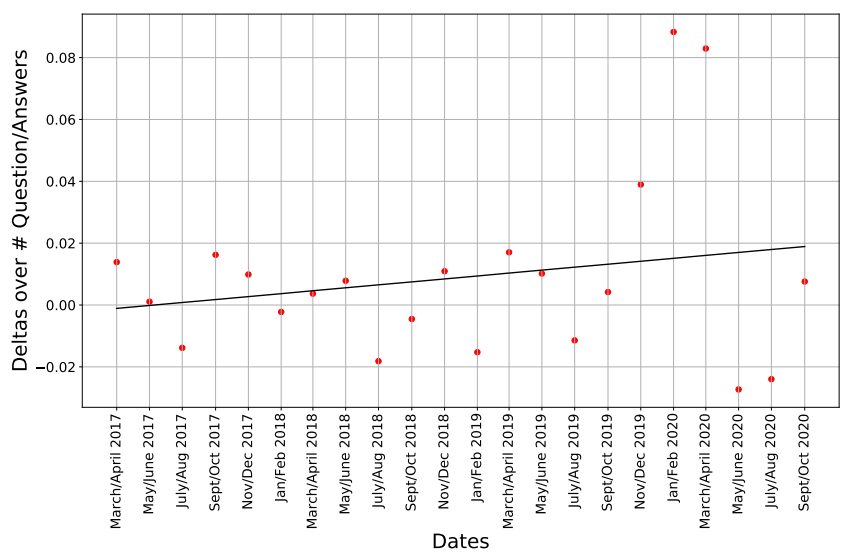

Figure 13: $\Delta$ over Bimonthly Ratio $Q / A$ on Stack Overflow

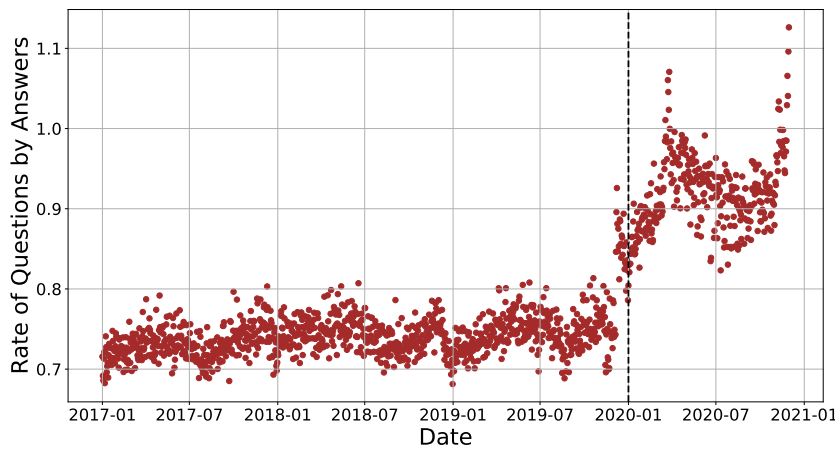

Figure 14: Ratio Questions/Answers on Stack Overflow

Table 8: RQ3: Statistics for Ratio Questions/Answers

\begin{tabular}{lrrrr}
\hline Year & 2017 & 2018 & 2019 & 2020 \\
\hline Mean & 0.73 & 0.74 & 0.75 & 0.92 \\
Var & 0.0004 & 0.0004 & 0.0013 & 0.0016 \\
Entropy & 0.86 & 0.86 & 0.77 & 0.93 \\
Outliers & 0 & 0 & 0 & 12 \\
\hline
\end{tabular}

differences between 2020 and the other years are all statistically significant. The numbers show that in 2020 the ratio $\mathrm{Q} / \mathrm{A}$ increased substantially, the entropy was also visibly higher, and the number of outliers was the largest ever. This shows, quite solidly, that in 2020 fewer answers were being provided in Stack Overflow with respect to the number of questions asked.

4.2.3 Comments and Votes. We also analyzed comments and votes, but, due to space limitations, we don't present the details of those analyses. The findings there were that there are no outliers, and we found nothing to be statistically significant to report. 
Table 9: Analysis of RQ's: Summary

\begin{tabular}{|c|c|c|c|c|c|c|c|}
\hline & \multirow{2}{*}{$\begin{array}{l}\text { GitHub } \\
\text { Push Ev. }\end{array}$} & \multicolumn{6}{|c|}{ Stack Overflow } \\
\hline & & Users & Q & A & $\mathrm{Q} / \mathrm{A}$ & Comments & Votes \\
\hline RQ1 & Yes & Yes & Yes & No & Yes & No & No \\
\hline RQ2 & No & No & No & No & Yes & No & No \\
\hline RQ3 & Yes/No & Yes/No & No & No & Yes & No & No \\
\hline
\end{tabular}

\section{DISCUSSION}

\subsection{Summary of findings}

Table 9 provides a summary of the results of all the research questions for both GitHub and Stack Overflow. When looking at GitHub, the only noticeably different characteristic of the 2020 push events data, as well as the other types of events that are highly correlated, is the number of outliers. Outliers, both positive and negative, represent unusually large increases/decreases in GitHub activity between two consecutive days. Specifically for push events, the two positive outliers in August and December were exceedingly out of line with the rest of the data. It would be interesting to further investigate what happened in those two days, but that falls out of scope of this study. As such, we cannot decisively conclude that the pandemic had an effect on people's activity in GitHub. Other studies of the effects of the COVID-19 on software development, using different methods, may explain the mixed signals. Looking just at Java repositories and based on developer surveys, Neto et al. [9] found that the impact of COVID-19 on developers has been mixed.

For Stack Overflow, as we discussed and see from the table, there has been a significant increase in the number of new users joining Stack Overflow and questions being posted there at the beginning of the pandemic (March/April 2020). Specifically, Stack Overflow experienced an unprecedented the number of new users joining the platform on March 31, 2020; more than 16,600 new users joined Stack Overflow on this day. The ratio Q/A also witnessed an increase in March/April 2020 as well as in Jan/Feb 2020.

We also observed that the number of answers, comments and votes experienced some increase during the beginning of the pandemic; the increase, however, was not significant. This leads to the assumption that at the beginning of the pandemic, developers started creating user accounts on Stack Overflow in order to be able to ask questions. They however, did not contribute to answering the questions, posting comments and voting on posts in the same rate as of questions.

When comparing the trends of activity in 2020 with previous years in RQ2 and RQ3, we observed differences in ratio Q/A in 2020 compared to all previous years combined and also, compared with each previous year; for all other activities, however, we did not observe any significant differences. As a result, although there has been a sudden peak in a group of Stack Overflow activities at the beginning of the pandemic, the overall activity was only different in terms of ratio Q/A in the year 2020 compared to other years. The other activities remained almost the same as before.

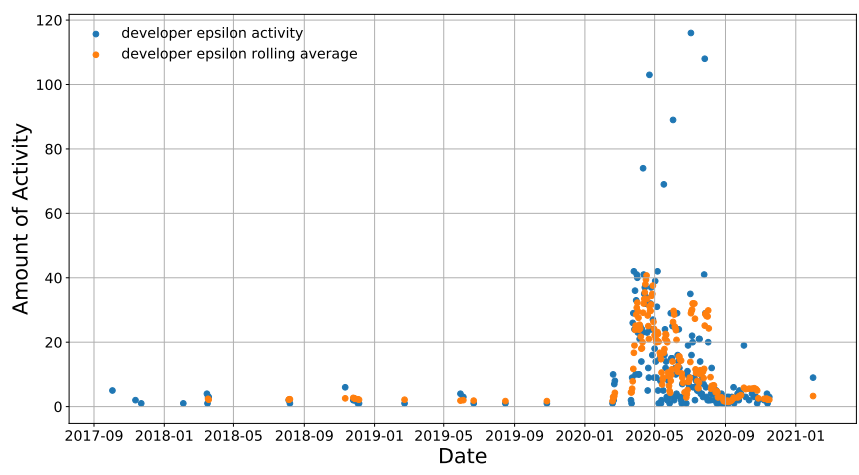

Figure 15: developer epsilon's activity

\subsection{Developer Testimonials}

Quantitatively looking at Figure 1 again, we see that there has been an increase in activity in Github during 2020. To have a deeper understanding of what happened during mid-2020, we sent out emails to twenty developers who had a noticeable peak in their activities during 2020, especially in the March/April time frame. A typical pattern of these unique contributions can be seen in Figure 15. Our email was very short, and did not constitute a formal survey; it simply contained a question: would you mind telling us if your increased contributions in March/April of last year were related to the pandemic, or were they unrelated?

We received seven replies, summarized in Table 10. Out of these seven developers, three of them reported the pandemic was the cause of their increased contributions, another three reported that the pandemic had nothing to do with their increased contributions, while another developer had mixed feelings. Our small sample size of responses is in line with Neto et al.'s work [9]. Nevertheless, it was interesting to find that three out of the seven developers who responded to our email attributed their increased activity directly to the pandemic.

\section{RELATED WORK}

Studies concerning developers and COVID-19 pandemic. Butler et al. [4] also analyzed the effects of pandemic and work from home on Microsoft employees. Their study revealed that developers faced challenges such as over-working, having many meetings and difficulty being motivated. On the other side of the spectrum, they logged some points of gratitude including more flexibility and being close to family. Xaverius [15] did a study on managing software development projects during COVID-19. Their study shows that managing a team virtually is different from managing it during the war against COVID-19. In another work, Rodeghero et al. [38] studied the on boarding experience of 267 people to software development teams at Microsoft during the pandemic, which showed that new hires faced challenges such as in building connections with their teammates. Ralph et al. [37] conducted a survey to assess the effects of COVID-19 pandemic on developers' productivity and well-being. Their study concluded the pandemic has negatively impacted developers' productivity and well-being and that women, 
Table 10: Summary of developer responses

\begin{tabular}{|c|c|}
\hline Id & Were your increased contributions $[. .$.$] due to the pandemic?$ \\
\hline alpha & "Yes". \\
\hline beta & $\begin{array}{l}\text { "The increase in contribution activity likely is unrelated to } \\
\text { the pandemic. I have started a new job during March } 2020 \text { which } \\
\text { wasn't as time consuming as my previous job". }\end{array}$ \\
\hline gamma & $\begin{array}{l}\text { "Hey, they were related to the pandemic } \\
\text { and growing increasingly unsatisfied with my main job". }\end{array}$ \\
\hline delta & $\begin{array}{l}\text { "My GitHub contributions didn't change much, in the sense, } \\
\text { that I might have similar pattern had there been no pandemic, } \\
\text { I might guess that I might even have more contributions } \\
\text { during this pandemic. It is a coincidence that my contributions } \\
\text { increased during this time frame". }\end{array}$ \\
\hline epsilon & $\begin{array}{l}\text { "Yes, my increase in contributions was related to the pandemic. } \\
\text { I got involved in an open-source group that } \\
\text { maintains a website tracking the Covid-19 cases in India". }\end{array}$ \\
\hline
\end{tabular}

Sorry, but my GitHub account is definitely an outlier that you should zeta exclude from such a study. Independently of COVID, I started to run lots of automated jobs that pushed to GitHub last year, so those explain the big increase jobs in $2020 "$.

"Pandemic kind of had both, good and bad impact on work. I was in fact a lot less productive during those days. But I also happened to get more time since I didn't have to go to college. For high contributions in March 2020, I would say the primary reason is, I ended up starting some new projects. I do feel I could've written a lot more code if it was a normal (non-pandemic) month of not having outside responsibilities".

parents and people with disabilities are being affected disproportionately. Russo et al. [39] studied how the onset of the COVID-19 pandemic disrupted the daily lives of software engineers. After conducting a longitudinal study, their results suggested that software engineers did activities they would do in the office at home. Also Microsoft [45] discusses the findings related to the pandemic's impact on work practices from an industry perspective. They show how the work practices have changed in several areas, from collaboration and meetings to IT and security, have changed. Bao et al. [3] did a quantitative analysis based on a dataset of developer's daily activities from Baidu Inc. They collected approximately 4,000 records of 139 developers' activities of 138 working days where $27 \%$ of the records are from when developers were working from home during the pandemic. Based on the results, they have found working from home has disadvantages and advantages on developer productivity and that productivity can vary for different developers.

Studies on GitHub. GitHub periodically publishes a report on the usage of their platform as The State of the Octoverse. Their 2020 Octoverse report [19] revealed that even though activity on GitHub has been steadily increasing over the years, 2020 was a year like no other and COVID-19 has had a strong effect on developer contributions. The exploratory data analysis reveals that developers are working longer, probably because they have difficulty finding a balance between work and play.
Neto et al. [9] mined 100 Java software repositories and surveyed the developers to understand the impact of COVID-19 on their well being. They also used different metrics to understand their results. The conclusion was that the findings regarding COVID-19 are on a spectrum. Wang et al. [47] studied COVID-19 themed projects on GitHub. They collected COVID-19 themed projects from GitHub . They form a systematic analysis to understand the development and maintenance behavior of COVID-19 themed repositories.This works demystifies the reaction of the OSS community to the pandemic. de Oliveira et al. [10] looked at a large quantity of questions from Stack Overflow and GitHub projects that aim to find trends that could help the community in the fight against COVID-19. Based on the questions from Stack Overflow and GitHub projects, a correlation has been identified between questions and projects during the pandemic.

Studies on Stack Overflow. Georgiou et al. [21] analyzed the Stack-Overflow platform in the context of COVID-19. Their study reveals that this global crisis has sparked an intense increase of activity on Stack Overflow with most of the topics reflecting an interest analyzing COVID-19 data.In our work, we try to determine, from a numerical stand point, whether there has has been a peak in the activities as the result of COVID-19 pandemic.

\section{LIMITATIONS AND THREATS TO VALIDITY}

We conducted this study by downloading Stack Overflow data from the internet archive and downloading GitHub data from GitHub Archive. Any errors in the data reported by these data sources can affect the results of this study. Another threat to validity of the GitHub analysis is the amount of automated bot activity. For many years, there has been bot activity in GitHub. We do not know whether bot activity had an unusual increase during 2020; our analysis assumes that the bot activity during 2020 followed whatever trends were already there before the pandemic.

\section{CONCLUSIONS}

We conducted a study to determine if COVID-19 impacted open source development. We mined GitHub data and Stack Overflow to see if development was affected due to the stay-at-home orders. When looking at GitHub, the only noticeably different characteristic of the 2020 events data is the number of outliers. We cannot decisively conclude that the pandemic had an effect on the increased activity in GitHub, although we identified some developers for whom it did. Factors from balancing work and domestic chores can influence productivity [22, 25, 42, 43]; indeed, we found some developers with increased contributions in 2020 who reported that the increase was not due to the pandemic. With Stack Overflow, we found out that there has been a significant increase on the number of new users and questions during the first months of the pandemic (March/April 2020). We also observed a large change in the ratio of Questions/Answers at the onset of pandemic as well a change in this ratio during the year 2020 compared to previous years, showing that developers were asking more questions but getting relatively fewer answers during that time.

\section{REFERENCES}

[1] Teresa Aguiar-Quintana, Thi Hong Hai Nguyen, Yasmina Araujo-Cabrera, and José M. Sanabria-Díaz. 2021. Do job insecurity, anxiety and depression caused by 
the COVID-19 pandemic influence hotel employees' self-rated task performance? The moderating role of employee resilience. International fournal of Hospitality Management 94 (2021), 102868. https://doi.org/10.1016/j.ijhm.2021.102868

[2] Matt Asay. 2020. COVID-19 isn't slowing open source-watch for developer burnout. https:/www.techrepublic.com/article/covid-19-isnt-slowing-opensource-watch-for-developer-burnout/

[3] Lingfeng Bao, Tao Li, Xin Xia, Kaiyu Zhu, Hui Li, and Xiaohu Yang. 2021. How does Working from Home Affect Developer Productivity? - A Case Study of Baidu During COVID-19 Pandemic. arXiv:2005.13167 [cs.SE]

[4] Jenna Butler and Sonia Jaffe. 2021. Challenges and Gratitude: A Diary Study of Software Engineers Working From Home During Covid-19 Pandemic. https://www.microsoft.com/en-us/research/publication/challenges-andgratitude-a-diary-study-of-software-engineers-working-from-home-duringcovid-19-pandemic/

[5] Emily Chen, Kristina Lerman, and Emilio Ferrara. 2020. Tracking Social Media Discourse About the COVID-19 Pandemic: Development of a Public Coronavirus Twitter Data Set. FMIR Public Health Surveill 6, 2 (29 May 2020), e19273. https //doi.org/10.2196/19273

[6] Elena Chong, Christopher Handscomb, Owain Williams, Robert Hall, and Michael Rooney. 2020. Agile resilience in the UK: Lessons from COVID-19 for the 'next normal'. https://www.mckinsey.com/business-functions/organization/our-insights/ agile-resilience-in-the-uk-lessons-from-covid-19-for-the-next-normal.

[7] Prithwiraj Choudhury, Wesley W Koo, and Xina Li. 2020. Working (from home) during a crisis: Online social contributions by workers during the coronavirus shock

[8] Rafael da Camara, Marcelo Marinho, Suzana Sampaio, and Saulo Cadete. 2020. How do Agile Software Startups deal with uncertainties by Covid-19 pandemic?

[9] Paulo Anselmo da Mota Silveira Neto, Umme Ayda Mannan, Eduardo Santana de Almeida, Nachiappan Nagappan, David Lo, Pavneet Singh Kochhar, Cuiyun Gao, and Iftekhar Ahmed. 2020. A Deep Dive on the Impact of COVID-19 in Software Development. arXiv:2008.07048 [cs.SE]

[10] Pedro Almir Martins de Oliveira, Pedro de Alcântara dos Santos Neto, Gleison Silva, Irvayne Ibiapina, Werney Lira, and Rossana Maria de Castro Andrade. 2021. Software Development During COVID-19 Pandemic: an Analysis of Stack Overflow and GitHub. arXiv:2103.05494 [cs.SE]

[11] Elizabeth Delahoussaye and AnnE Rice. 2020. Working from Home During COVID-19: Challenges and Solutions. https://journal.ahima.org/working-fromhome-during-covid-19-challenges-and-solutions/

[12] Isaure Delaporte and Werner Pena. 2020. Working from home under Covid-19: Who is affected? Evidence from Latin American and Caribbean countries.

[13] Michael Dockery and Sherry Bawa. 2020. Working from Home in the COVID-19 Lockdown

[14] Yadolah Dodge. 2008. The Concise Encyclopedia of Statistics. https://doi.org/10. 1007/978-0-387-32833-1

[15] Yadolah Dodge. 2020. Manage Software Development Project Using Virtual Teams During COVID-19. https://papers.ssrn.com/sol3/papers.cfm?abstract_id=3591096. Accessed: 2020-01-11.

[16] Adrian Fernandez-Perez, Aaron Gilbert, Ivan Indriawan, and Nhut H. Nguyen 2021. COVID-19 pandemic and stock market response: A culture effect. Fournal of Behavioral and Experimental Finance 29 (2021), 100454. https://doi.org/10. 1016/j.jbef.2020.100454

[17] Denae Ford, Margaret-Anne Storey, Thomas Zimmermann, Christian Bird, Sonia Jaffe, Chandra Maddila, Jenna L. Butler, Brian Houck, and Nachiappan Nagappan. 2020. A Tale of Two Cities: Software Developers Working from Home During the COVID-19 Pandemic. arXiv:2008.11147 [cs.SE]

[18] Tom Foremski. 2020. Stack Overflow reports strong growth from COVID-19 workplace changes. https://www.zdnet.com/article/stack-overflow-reportsstrong-growth-from-covid-19-workplace-changes/

[19] Nicole Forsgren. 2020. The 2020 State of the OCTOVERSE. https://octoverse github.com/. Accessed: 2021-01-11.

[20] James H. Fowler, Seth J. Hill, Remy Levin, and Nick Obradovich. 2020. The effect of stay-at-home orders on COVID-19 cases and fatalities in the United States. arXiv:2004.06098 [stat.AP]

[21] Konstantinos Georgiou, Nikolaos Mittas, Lefteris Angelis, and Alexander Chatzigeorgiou. 2020. A Study of Knowledge Sharing related to Covid-19 Pandemic in Stack Overflow. arXiv:2004.09495 [cs.SE]

[22] ADAM Gorlick. 2020. The productivity pitfalls of working from home in the age of COVID-19. Stanford News. March 30 (2020), 2020.

[23] Abid Haleem, Mohd Javaid, and Raju Vaishya. 2020. Effects of COVID-19 pandemic in daily life. Current Medicine Research and Practice 10, 2 (2020), 78-79. https://doi.org/10.1016/j.cmrp.2020.03.011

[24] Sherrill Hayes, Jennifer L Priestley, Namazbai Ishmakhametov, and Herman E Ray. 2020. "I'm not Working from Home, I'm Living at Work": Perceived Stress and Work-Related Burnout before and during COVID-19.

[25] Mirza Waseem Hussain, Tabasum Mirza, and Malik Mubasher Hassan. 2020 Impact of COVID-19 Pandemic on the Human Behavior [J]. International fournal of Education and Management Engineering 10, 8 (2020), 35-61.
[26] Marijn Janssen and Haiko van der Voort. 2020. Agile and adaptive governance in crisis response: Lessons from the COVID-19 pandemic. International fournal of Information Management 55 (2020), 102180. https://doi.org/10.1016/j.ijinfomgt. 2020.102180 Impact of COVID-19 Pandemic on Information Management Research and Practice: Editorial Perspectives.

[27] Ceilla Amanda Jasmine. 2019. Impacts of Covid-19 on Company and Efforts to Support Organization Adaptable. , 67-70 pages.

[28] JRebel. 2020. COVID-19 Impact on Developer Productivity. https://www.jrebel. com/blog/covid-19-impact-developer-productivity

[29] Tae Kim. 2015. T test as a parametric statistic. Korean fournal of Anesthesiology 68 (11 2015), 540. https://doi.org/10.4097/kjae.2015.68.6.540

[30] Nick Kolakowski. 2020. GitHub shows COVID-19 SHIFTING developer workflows. https://insights.dice.com/2020/05/07/github-shows- covid-19-shiftingdeveloper-workflows/

[31] Weipeng Lin, Yiduo Shao, Guiquan Li, Yirong Guo, and Xiaojun Zhan. 2021 The psychological implications of COVID-19 on employee job insecurity and its consequences: The mitigating role of organization adaptive practices. Fournal of Applied Psychology 106, 3 (2021), 317.

[32] Albert Madansky. 1988. Testing for Normality. Springer New York, New York, NY, 14-55. https://doi.org/10.1007/978-1-4612-3794-5_2

[33] Dennis Mancl and Steven D. Fraser. 2020. COVID-19's Influence on the Future of Agile. In Agile Processes in Software Engineering and Extreme Programming Workshops, Maria Paasivaara and Philippe Kruchten (Eds.). Springer International Publishing, Cham, 309-316.

[34] Rani Molla. 2021. 10 ways office work will never be the same. https://www.vox. com/recode/22331447/10-ways-office-work-pandemic-future-remote-work

[35] David C Ng, Seedahmed S Mahmoud, Eric S Hald, and Qiang Fang. 2020. Overcoming challenges in teaching calculus remotely during COVID-19 pandemic.

[36] A. Nielsen. 2019. Practical Time Series Analysis: Prediction with Statistics and Machine Learning. https://books.google.com/books?id=uq0avgEACAAJ

[37] Paul Ralph, Sebastian Baltes, Gianisa Adisaputri, Richard Torkar, Vladimir Kovalenko, Marcos Kalinowski, Nicole Novielli, Shin Yoo, Xavier Devroey, Xin Tan, and et al. 2020. Pandemic programming. Empirical Software Engineering 25, 6 (Sep 2020), 4927-4961. https://doi.org/10.1007/s10664-020-09875-y

[38] Paige Rodeghero, Thomas Zimmermann, Brian Houck, and Denae Ford. 2021. Please Turn Your Cameras On: Remote Onboarding of Software Developers during a Pandemic. , 41-50 pages. https://doi.org/10.1109/ICSE-SEIP52600.2021. 00013

[39] Daniel Russo, Paul P. H. Hanel, Seraphina Altnickel, and Niels van Berkel. 2021. The Daily Life of Software Engineers during the COVID-19 Pandemic. arXiv:2101.04363 [cs.SE]

[40] Jenna Sargent, About Jenna Sargent Jenna Sargent is an Online, Social Media Editor for SD Times. She covers Microsoft, Jenna Sargent is an Online, Social Media Editor for SD Times. She covers Microsoft, and View all posts by Jenna Sargent. 2020. Developers take on COVID-19 with open-source projects, hackathons. https://sdtimes.com/open-source/developers-take-on-covid-19with-open-source-projects-hackathons/

[41] Sarah Schlothauer, Author Sarah Schlothauer All Posts by Sarah Schlothauer Sarah Schlothauer is the editor for JAXenter.com. She received her Bachelor's degree from Monmouth U, Sarah Schlothauer All Posts by Sarah Schlothauer, All Posts by Sarah Schlothauer, and Sarah Schlothauer is the editor for JAXenter.com. She received her Bachelor's degree from Monmouth University. 2020. COVID-19 vs open source: How developers are fighting the virus. https://jaxenter.com/ covid-19-open-source-170237.html

[42] Darja Smite, Nils Brede Moe, Eriks Klotins, and Javier Gonzalez-Huerta. 2021. Work Patterns of Software Engineers in the Forced Working-From-Home Mode. arXiv:2101.08315 https://arxiv.org/abs/2101.08315

[43] Jayne Smith. 2020. Several factors impact wellbeing of people working from home. https://workplaceinsight.net/covid-19-just-one-factor-impactingwellbeing-of-employees-working-from-home/

[44] Daniel Spurk and Caroline Straub. 2020. Flexible employment relationships and careers in times of the COVID-19 pandemic.

[45] Jaime Teevan, Brent Hecht, Sonia Jaffe, Nancy Baym, Rachel Bergmann, Matt Brodsky, Bill Buxton, Jenna Butler, Adam Coleman, Mary Czerwinski, Brian Houck, Ginger Hudson, Shamsi Iqbal, Chandra Maddila, Kate Nowak, Emily Peloquin, Ricardo Reyna Fernandez, Sean Rintel, Abigail Sellen, Tiffany Smith, Margaret-Anne Storey, Siddharth Suri, Hana Wolf, and Longqi Yang. 2021. The New Future of Work: Research from Microsoft into the Pandemic's Impact on Work Practices. Technical Report MSR-TR-2021-1. Microsoft. https: //www.microsoft.com/en-us/research/publication/the-new-future-of-workresearch-from-microsoft-into-the-pandemics-impact-on-work-practices/

[46] Liu Wang, Ruiqing Li, Jiaxin Zhu, Guangdong Bai, and Haoyu Wang. 2020. When the Open Source Community Meets COVID-19: Characterizing COVID-19 themed GitHub Repositories. arXiv:2010.12218 https://arxiv.org/abs/2010.12218

[47] Liu Wang, Ruiqing Li, Jiaxin Zhu, Guangdong Bai, and Haoyu Wang. 2020. When the Open Source Community Meets COVID-19: Characterizing COVID-19 themed GitHub Repositories. arXiv:2010.12218 [cs.SE] 
[48] Rand Wilcox. 2001. Fundamentals of Modern Statistical Methods: Substantially Improving Power and Accuracy. https://doi.org/10.1007/978-1-4419-5525-8 